\title{
Cardiovascular prevention and rehabilitation for patients with ventricular assist device From exercise therapy to long-term therapy Part I: exercise therapy
}

\author{
Prevenzione e riabilitazione cardiovascolare \\ in pazienti con device di assistenza ventricolare \\ Dall'esercizio alla terapia a lungo termine \\ Parte l: esercizio
}

\author{
Ugo Corrà1, Massimo Pistono1, Alessandro Mezzani1, Marco Gnemmi1, \\ Franco Tarro Genta2, Roberto Caruso1, Pantaleo Giannuzzi1
}

\begin{abstract}
Cardiovascular prevention and rehabilitation for patients with ventricular assist device. From exercise therapy to long-term therapy. Part I: exercise therapy. U. Corrà, M. Pistono, A. Mezzani, M. Gnemmi, F. Tarro Genta, R. Caruso, P. Giannuzzi.

In the present context of an aging population, limited donor heart availability, improved reliability of mechanical cardiac support and improved patient outcomes, ventricular assist device (VAD) options to support end-stage heart failure patients are rapidly expanding. In addition, both the smaller size and lighter weight of the pumps now produced and early evidence that these third generation devices may be associated with lower risk of infection and right ventricular failure will probably lead to greater physician and patient acceptability.

This is the first of a two-part review on the role of cardiovascular prevention and rehabilitation in patients
\end{abstract}

with VAD. In this first part, we will discuss the role of exercise therapy in VAD patients, while the second will focus on long-term management. One of the prerequisites for use of a VAD - whether permanent (as destination therapy) or semi-permanent (as an alternative to heart transplantation) - is that exercise capacity, although not normal, must be adequate for daily life activities. An intensive multidisciplinary rehabilitation program has the potential to increase exercise performance and improve the quality of life of VAD patients. Both early progressive mobilization and exercise training may improve the overall condition of VAD patients, and favorably impact their clinical course.

Key words: rehabilitation, left ventricular assist device.

Monaldi Arch Chest Dis 2011; 76: 27-32.

1 Division of Cardiology, Salvatore Maugeri Foundation, IRCCS, Veruno (NO), Italy.

2 Division of Cardiology, Clinica Major, Salvatore Maugeri Foundation, Torino, Italy.

Corresponding author: Dr. Ugo Corrà; Divisione di Cardiologia, Laboratory for the analysis of cardio-respiratory signals; IRCCS Fondazione "S. Maugeri"; Via per Revislate, 13, I-28010 Veruno (NO), Italy; Phone: 0039-0322-884711; Fax: 0039-0322-830294; ugo.corrà@fsm.

Although cardiac rehabilitation has undergone a profound transformation since it first appeared in the 1960 s, with modern programs now embracing both secondary prevention activities and high risk situations such as chronic heart failure (HF) and implanted cardiac devices (pacemaker, ventricular resynchronisation, implantable cardioverter defibrillators) [1-2], therapeutic exercise still remains a core component. Important goals of cardiac rehabilitation and secondary prevention programs are to increase functional capacity, decrease symptoms, promote psychosocial well-being, and facilitate the return to work. In this perspective, patients with mechanical circulatory support (MCS) are natural candidates for referral to contemporary cardiac rehabilitation and secondary prevention programs.
Reliability of mechanical circulatory support (MCS): an expanding scenario

For many years, heart transplantation (HT) was the only proven therapy for end-stage heart failure (HF), but it has been, and for the foreseeable future will continue to be, severely limited by the availability of donor hearts [3]. MCSs substitute the circulatory function of one or both sides of the heart, and have revolutionized the treatment of advanced $\mathrm{HF}$ patients [4]. Different devices are currently available, and a brief description of MCSs is in order before we focus on the exercise-related issues in these patients.

The biomechanics of blood flow generated by MCS is relatively straightforward [4]: an inflow cannula is connected to a pump, which is then con- 
nected to an outflow cannula. Blood flow is generated in either a pulsatile or continuous flow, depending on the specific device, and circulatory support can be provided with a left ventricular assist device (LVAD), right ventricular assist device (RVAD), or biventricular assist device (BiVAD). Three categories of MCS patients have be described [5]: 1) individuals who require temporary circulatory support who are expected to recover after cardiac injury and will not need HT (bridge to recovery); 2) patients awaiting HT but who would not survive the wait until an organ is available owing to low cardiac output and/or noncardiac comorbidities (bridge to transplantation); 3) individuals who need long-term support but have a relative or absolute contraindication to HT (destination therapy, DT). The 3rd Interagency Registry for Mechanical Assisted Circulatory Support (INTERMACS) Annual Report [6] has documented a transition from pulsatile-flow to continuous-flow pumps, and an increase of patients implanted as DT, associated with an improvement in medium-term survival.

Summary remarks: at present, it seems realistic that MCS will become an alternative to HT, but one of the prerequisites for permanent or semi-permanent VAD use is that exercise performance, although not normal, must be adequate for daily life activities: for this reason, cardiologists in charge of MCS patients must well trained in exercise physiology, exercise testing, and exercise training.

\section{Exercise hemodynamics}

LVAD recipients demonstrate improvement in exercise tolerance, non-cardiac organ function, as well as metabolic and neurohormonal levels [7-9]. The positive effects of LVAD on left ventricular (LV) function include marked reduction in LV systolic and diastolic pressures, leading to reverse LV remodeling, a normalized pressure volume relation, and myocyte recovery, with improved contractile function. At rest, the acute effects of LVAD consist of a decrease in resting left and right atrial pressures and an increase in cardiac index, vascular conductance and blood flow [4-5, 8]. Resting hemodynamic changes occur immediately after implantation, in most cases within less than 2 days.

Virtually all LVAD recipients should be physically active. When challenged with any exercise task, the human body responds through a series of integrated changes in function that involve most, if not all, of its physiologic systems. The magnitude of these changes depends largely on the intensity and duration of the exercise sessions, the force or load used, and the body's initial level of fitness. For LVAD patients, apart from the level of their disease severity, the design itself of the device is also important. As mentioned, LVADs can be broadly categorized as displacement pulsatile or rotary continuous flow [10]. Pulsatile are volume displacement devices that operate in a full-toempty mode that automatically adjusts beat rate to changes in LV preload; the new generation LVADs incorporate continuous-flow rotary pump technology with axial configuration. Haft et al. [11] evaluated exercise performance in two groups of patients according to LVAD design: Heart-Mate (HM) XVE, a pul- satile device, vs. HM II, a continuous-flow rotary design. Briefly, the HM XVE is typically operated in a full-to-empty mode: beat rate automatically increases or decreases in response to changes in LV preload and filling rate of the pump chamber. Maximum device output is approximately $10 \mathrm{~L} /$ minute at a heart rate of 120 beats/minute. The HM II (Thoratec, Inc) is a continuous-flow rotary pump with an axial design: the pump has an operating RPM range of 6000 to 15000 and can generate up to $10 \mathrm{~L} / \mathrm{minute}$ of flow at a pressure of $\sim 100 \mathrm{~mm} \mathrm{Hg}$. Haft et al.showed that peak oxygen consumption (VO2) was similar for the two groups at 3 months after LVAD implantation (15.4 \pm 4.0 for HM XVE vs. $15.6 \pm 4.7 \mathrm{~mL} / \mathrm{kg} / \mathrm{min}$. for HM II), despite significant differences in LV volume unloading: decrease in LV size, reduction in degree of mitral insufficiency, and increase in LV ejection fraction were significantly greater for the HM XVE group. These results confirmed those by Jaski et al. [12]: no differences in peak VO2 were observed between patients with two different LVAD designs, both pulsatile devices but with different mechanisms of device actuation (HM IP-pneumatic; HM VE-electrical).

The function of the native ventricle is important for the exercise physiology of LVAD. The native heart-LVAD complex responds physiologically and demonstrates a significant circulatory reserve consistent with the capability to meet demands of daily activities [13]. Jaski et al. [14] showed that patients with an asynchronous fill to empty mode LVAD can increase cardiac output (CO) by increasing rate with increased venous return: LVAD stroke volume was set and remained essentially constant, much as in normal hearts, where stroke volume changes only slightly to achieve the increased $\mathrm{CO}$ during vigorous exercise. Most if not all of the systemic CO at rest was contributed by the LVAD, and minimal to no aortic valve opening or left ventricular outflow tract ejection was documented: on the contrary, during dynamic exercise, aortic ejection was apparent and total systemic $\mathrm{CO}$ exceeded the LVAD output. Therefore, although the LVAD is designed to function parallel, in effect it works in series with the left ventricle.

Summary remarks: in LVAD recipients, exercise is a complex, composite integration between the physiologic systems (altered by the disease) and the device function [13-15]: exercise performance is the result of numerous adaptive mechanisms that involve the native $\mathrm{LV}$, the right ventricle (RV), the pulmonary hemodynamic, the overall and muscle conditioning, and the device. In addition, several non hemodynamic- or device-related factors, such as duration of HF symptoms, preoperative length of hospital and intensive care unit stay, preoperative serum prealbumin, duration of postoperative recovery, and medical therapy, may all affect exercise tolerance as well. Of note, dissimilarities in LVAD designs seem to play a marginal role.

\section{Early mobilization}

Ideally, LVAD recipients should initiate an exercise program as soon as possible [16]. The ultimate goals of early mobilization are to prevent postoperative complications due to bed rest, minimize loss of mobility, maximize independence, and facilitate 
weaning from the ventilator [16-17]. As a general rule, early mobilization should take into account the pre-LVAD condition: some patients with acute hemodynamic instability and/or recent HF may already be familiar with exercise modes and training sessions, and able to reinitiate the training program promptly with minimal reeducation. On the other hand, patients who were dependent on long-term inotropic support therapy or who had signs or symptoms of deconditioning might need a slower, more cautious approach. Nevertheless, in the immediate post-LVAD implantation period, a series of sequential steps needs to be followed [17]: the patient should first be evaluated by a physical therapist (Table 1) and an individualized treatment plan of physical therapy defined (Table 2). Since physical therapy interventions are dependent on overall medical condition and hemodynamic stability, complications (e.g. infections, RV failure, bleeding, ventricular arrhythmia, anorexia and neurological impediments) can postpone the start of physical therapy or delay its course.

\section{Table 1. - Physical therapy pre-evaluation}

Detailed review of recent and past medical history.

Review of prior level of function.

Mental status, and cognitive ability.

Vital signs, screening for cardiovascular instability.

Medications, i.e. need for continuous or intermittent infusions (inotropic drugs).

Ventilator settings or oxygen requirements.

Surgical wound, and skin integrity.

Range of motion, coordination, balance, strength, endurance.

Functional assessment:

1. Bed mobility.

2. Transfers.

3. Gait.

4. Activities of daily living.

Table 2. - Individualised physical therapy interventions for early mobilization

\section{Positioning}

Exercises

- Muscle strengthening.

- Breathing.

Bed mobility activities

- Sitting on edge of bed, in association with exercises, trunk control.

- Turning side to side.

Transfers from bed to

- Stretcher-chair.

- Chair.

- Commode.

Gait

- Pre-gait activities: weight shifting, stepping in place and sideways.

- Gait training with rolling walker.
Therefore, LVAD patients should be closely monitored for any signs of exercise-related complications or LVAD dysfunction alarms (Table 3). If such occur, the physical therapy session should be interrupted, and re-initiated when clinical condition and/or device operability are restored. An open approach is recommended, and the physical therapist should liaise closely with physicians, nurses, respiratory therapists and psychologists to ensure efficient and safe mobilization.

Exercise should consist mainly of passive and active range of motion accompanied by incentive spirometry to facilitate pulmonary toilet. Once out of bed in a chair, leg raising and hip girdle exercises are useful as a preparation to transfer weight from sitting to standing. Once the patient is able to stand, ambulation should be initiated, initially in the patient's room, progressing later to the ward. Later on, a set routine consisting of bicycle, treadmill, upper body ergometry and free weights can be carried out safely in the controlled intensive rehabilitation setting. Table 4 presents early mobilization exercise

Table 3. - Criteria for termination of physical therapy session

Significant drop in LVAD flow or suction alarm.

Hypotension associated with fainting, dizziness, or diaphoresis.

Supine resting heart rate $>100$ beats per minute.

Severe, intolerable dyspnea.

Saturation less than $90 \%$ on supplemental oxygen.

Significant chest pain or discomfort.

Extreme fatigue or claudicatio.

Request of patient to stop.

$>1.8 \mathrm{Kg}$ increase in body mass over previous 1 to 3 days.

Complex ventricular arrhythmia at rest or appearing with exertion.

Table 4. - Early mobilization-exercise modalities based on current published literature

Move all extremities in sitting position.

Sit in chair.

Move all extremities in standing position.

Ambulate with assistance.

Full range of motion.

Progressive ambulation.

Walk on level surface.

Treadmill walking.

Independent ambulation.

Begin stair climbing.

Lifting small weights.

Arm ergometry.

Cycling and treadmill walking. 
modalities based on the current literature [18], to be carried out in a 6 -week rehabilitation program. The frequency of sessions is once a day, 6-7 days per week, with a duration varying from 15 minutes to 1 hour, as tolerated.

Summary remarks: as is often the case for cardiovascular diseases, early mobilization is recommended; however the severity and heterogeneity of clinical manifestations, differences in medical prescriptions and concurrent complications preclude any standardization. Modalities of interventions, timing and duration of mobilization differ according to the individual patient, and cannot be generalized.

\section{Exercise performance}

The poor exercise performance and quality of life (QoL) scores despite optimal medical and electrical therapies, including cardiac resynchronization therapy when appropriate, highlight the limitations of currently utilized treatments for patients with end-stage HF. Thus, a critical therapeutic goal is to enhance patients' QoL and functional capabilities. LVADs significantly affect NYHA class status, QoL, peak aerobic capacity and submaximal exercise tolerance. An early and sustained clinically meaningful improvement in NYHA status, QoL and distance at the 6-minute walk (6MWD) test has been clearly demonstrated [9, 16, 19-22]. In 103 patients, with pulsatile or continuous flow HM, mean 6 MWD was $393 \pm 290$ meters, approaching the lower range for patients without cardiovascular disease (400 meters), while, as regards QoL, the mean Minnesota Living with Heart Failure Questionnaire (MLHFQ) score was 35 \pm 31 , which correlates with NYHA Class I to II: of note, no differences in 6MDW and QoL were observed if patients were stratified by LVAD type.

A substantial increase in peak oxygen consumption (VO2) has been reported [22-24) within a few months after VAD implantation. A range of peak VO2 from 14 to $24 \mathrm{ml} / \mathrm{kg} / \mathrm{min}$ has been described; however, across studies, the work load of most of LVAD patients remains sub-optimal, i.e. about $50 \%$ to $60 \%$ of what is predicted for a given age and gender [13].

Exercise capacity post-LVAD implantation has been compared to that of advanced HF patients and HT recipients. Peak VO2 of LVAD (TCI HM, Thermo Cardiosystems, Inc) patients was significantly greater than that of severe HF patients, with a mean peak $\mathrm{VO} 2$ of $12 \pm 3.0 \mathrm{~mL} / \mathrm{kg} / \mathrm{min}$. [23]: since medical treatment of LVAD patients was minimal, it was claimed that the level of exercise performance was due almost entirely to the device therapy. Importantly, although LVAD patients showed a significantly greater $\mathrm{CO}$ at rest and peak exercise (LVAD output increased from $\sim 5 \mathrm{~L} / \mathrm{min}$ at rest to $11.2-11.4$ $\mathrm{L} / \mathrm{min}$ at peak exercise), pulmonary pressures rose significantly with exercise both in HF patients and LVAD recipients. However, even though mean pulmonary artery pressure and pulmonary capillary wedge pressure were significantly lower in the LVAD patients at peak exercise [23], the slope of minute ventilation vs. carbon dioxide production (VE/CO2 slope) was similar between the two groups, suggesting that the relief of pulmonary hypertension achieved with LVAD therapy had no impact on the ventilatory response to exercise. This finding is consistent with the observation that acute vasodilation does not affect the ventilatory response in patients with chronic HF [25].

LAVD patients had a lower peak VO2, peak predicted VO2, and a shorter treadmill exercise duration 1 to 3 months after implantation compared to a similar time post-HT [12]: peak VO2 correlated with peak LVAD rate and output. Kugler et al. [22] showed that physical exercise tolerance increased in both the HT and LVAD groups, a result most likely attributable to the benefits of the surgical procedure; however, both groups had sub-optimal exercise tolerance despite optimal treatment. After adjusting for age, gender and BMI, the LVAD group had a lower exercise tolerance compared to the HT group [22]. De Jonge et al. [24] evaluated exercise performance, with a sequential analysis, in two small LVAD and HT groups: in the first group, exercise performance was studied 8 and 12 weeks after LVAD implantation, while, in the second group, exercise capacity was evaluated in patients who underwent, first, LVAD implantation and then HT: exercise testing was performed 12 weeks after each procedure. All patients (first and second group) were mobilized at an early stage and participated in an intensive, supervised rehabilitation program based on dynamic exercise, strength and endurance training. In the first group, a significant increase in peak VO2 (from $21.3 \pm 3.8$ to $24.2 \pm 4.8 \mathrm{ml} / \mathrm{kg} / \mathrm{min}$ ) accompanied by a significant decrease in VE/VCO2 $(39.4 \pm 10.1$ to $36.3 \pm 8.2$ ) was observed at 8 weeks. In the second group, similar peak $\mathrm{VO} 2, \mathrm{VO} 2$ at the anaerobic threshold and VE/VCO2 values were observed 12 weeks after LAVD and after HT.

Summary remarks: in LVAD recipients, functional capacity is considerably improved compared with the pre-implantation moribund condition, and longer assist times may result in an even better exercise performance. Functional capacity can diverge because of different postoperative care and convalescence, but, apart from the limitations imposed by the operating console and the drive line, exercise capacity and daily routine activities are expected to be comparable to those of HT patients, and superior to those of advanced HF ones. Nonetheless, even though exercise testing cannot be performed in some LVAD recipients because of critical illness and complications, and hence the evaluation of only the "happy" survivors or those spared from severe comorbidity might result in an overestimation of true exercise tolerance, the exercise performance remains sub-optimal. This suggests a need not only for longterm exercise rehabilitation programs with targeted interventions on physical and psychosocial functioning, but also a modification of focus from survival to improved coping with illness-related requirements.

\section{Exercise training}

As regards exercise training (ET), assessment in LVAD recipients is scant. Data on content, setting, duration of the sessions and safety are vague, and consequently it is unclear how progressive long- 
term exercise programs should be set up. Even if, up to now, only anecdotal and case reports have been reported, a formal exercise training, usually in the form of progressive ambulation, bicycle, or treadmill exercise, should be started after hemodynamic stability is achieved. In addition, the basic principles of exercise prescription (frequency, intensity, duration, and mode) are applicable for LVAD recipients, as well [18]. Mettauer et al. [26] performed ET in a single patient, 3 weeks after LVAD (HeartMate 1000 IP) implantation. ET consisted of 20-30 min of constant rate stationary bicycle exercise daily, with the work rate set to obtain $50 \%$ of the maximal heart rate increase recorded during the incremental exercise test: VO2 increased by $64 \%$ at peak exercise and by $56 \%$ at the anaerobic threshold after 6 weeks of ET. Of note, further LV unloading, and increased RV load at peak exercise, leading to a decreased RV ejection fraction was observed after ET: finally, neuro-hormonal drive decreased for a given work load. Makita et al. [27] measured the anaerobic threshold levels during cardiopulmonary exercise tests in 9 LVAD patients. A training program of 10$30 \mathrm{~min}$ of bicycle exercise at the anaerobic threshold level 2-3 times a week plus walking in the ward on other days led to a significant increase in peak work rate. De Jonge et al. [24] proposed an interval training program for LVAD recipients: initially, the training regimen consisted of 2 to 6 min of low-level activities alternated with 1 to $2 \mathrm{~min}$ of rest. Training included sessions on the bicycle and treadmill, as well as with the rowing machine. Gradually, intensity was adjusted according to the level of perceived exertion ( 2 to 4 corresponding to "light" to "somewhat hard") on the Borg scale of 0 to 10 , with exertional dyspnea not exceeding 2 on a dyspnea scale of 0 to 4 . Duration of exercise was progressively increased to 20 to $40 \mathrm{~min} /$ day three to five times a week. In addition, strength and endurance training of local muscle groups was performed. Unfortunately, tolerability and acceptability of this intensive post-operative exercise rehabilitation program were not reported.

Summary remarks: exercise training in LVAD is in its infancy. Although data are rapidly accumulating, several questions remain unsolved and the interpretation of LVAD adaptation to exercise sessions is complex. For instance, in the same patient, at the beginning of the exercise session one might observe a change in pump flow volume as a result of the change in posture, while the device output thereafter remains steady. Changes in pump flow might be either symptomatic or asymptomatic. In addition, since both the LVAD output and the patient's native LV output contribute to the total output during exercise, and individual differences in physiologic and LVAD responses to exercise have been observed, an individual-based regulation of LVAD function should be considered to maximize the effectiveness of the device for an efficient and safe ET program.

\section{Conclusions and future directions}

As the impact of device therapy continues to increase and permeate the medical community, it is imperative to disseminate knowledge and experi- ences. LVAD recipients show a significant exercise improvement after implantation, but their functional capacity remains sub-optimal. There is room for improvement, and there is an astounding opportunity for cardiac rehabilitation to determine the most effective ways to promote and standardize specific exercise regimes, targeted at both physical and psychosocial functioning, and to motivate LVAD patients to practise a level of physical activity that can benefit their health and well-being. The challenge that lies ahead is formidable.

Aknowledgment: The authors are grateful to Rosemary Allpress for her careful revision of the English manuscript.

We are in special debt to the late Dr Enzo Bosimini who, as the first of our team in charge of managing patients with mechanical circulatory support, passed on to us his knowledge and expertise, stimulated our research curiosity, and created the existing platform for us to work together as a team.

In addition, we would like to thank Prof. Giuseppe Faggian and Dr Alberto Forni of Cardiochirurgia Universitaria, Ospedale Civile Maggiore di Verona Borgo Trento, Italy; Prof. Mauro Rinaldi and Dr. Paolo Centofanti of Cardiochirurgia Universitaria, Ospedale S. Giovanni Battista di Torino, Italy; Dr. Federico Pappalardo of the Rianimazione Cardiochirurgica, and Dr. Michele De Bonis of the Cardiochirurgia, IRCCS San Raffaele Milano, for their effective and most valuable collaboration.

Finally, we are obliged to Ing Silvia Scuri, Dr. Emilio Contini (Artech srl, "Jarvik Italia"), Dott. Annalisa Rastelli, Dott. Mauro Cotza ("THORATEC - Hear Mate Italia"), Ing. Antonella Marino, and Claudio Gibelli (Vega srl, "Berlin Hear Italia") for their passionate technical support.

\section{References}

1. Piepoli MF, Corrà U, Benzer W, et al. Secondary prevention through cardiac rehabilitation: from knowledge to implementation. A position paper from the Cardiac Rehabilitation Section of the European Association of Cardiovascular Prevention and Rehabilitation. Eur J Cardiovasc Prev Rehabil 2010, 17: 1-17.

2. Saner H, Wood D. Practical organisation of preventive cardiology programmes: integrating prevention and rehabilitation. Eur J Cardiovasc Prev Rehabil 2009, 16 (Suppl 2): S37-S42.

3. Costanzo MC, MD Task Force 1, Taylor D, MD Task Force 2, Sharon Hunt S, MD Task Force 3, on behalf of the International Society for Heart and Lung Transplantation (ISHLT). The International Society of Heart and Lung Transplantation Guidelines for the care of heart transplant recipients. J Heart Lung Transplant 2010; 29: 914-956.

4. Hiestand BC, Circulatory assist devices in heart failure patients. Heart Failure Clin 2009; 5: 55-62.

5. Stevenson LW, Rose EA, Left Ventricular Assist Devices Bridges to Transplantation, Recovery, and Destination for Whom?. Circulation 2003; 108: 3059-3063.

6. Interagency Registry for Mechanically Assisted Circulatory Support (INTERMACS). Available at: http:// www.intermacs.org/membership.aspx. Accessed February 28, 2011.

7. Bank AJ, Mir SH, Nguyen DQ, et al. Effects of left ventricular assist devices on outcomes in patients undergoing heart transplantation. Ann Thorac Surg 2000; 69: 1369-1374.

8. Jaski BE, Lingle RJ, Reardon LC, Dembitsky WP. Left ventricular assist device as a bridge to patient and myocardial recovery. Prog Cardiovasc Dis 2000; 43: 5-18. 
9. Levin HR, Chen JM, Oz MC, et al. Potential of left ventricular assist devices as outpatient therapy while awaiting transplantation. Ann Thorac Surg 1994; 58: 1515-1520.

10. Griffith BP, Kormos RL, Borovetz HS, et al. HeartMate II left ventricular assist system: from concept to first clinical use. Ann Thorac Surg 2001; 71: 116-120.

11. Haft J, Armstrong W, Dyke DB, et al. Hemodynamic and exercise performance with pulsatile and continuous-flow left ventricular assist devices. Circulation 2007; 116 [suppl I]: I-8-I-15.

12. Jaski BE, Lingle RJ, Kim J, et al. Comparison of functional capacity in patients with end-stage heart failure following implantation of a left ventricular assist device versus heart transplantation: results of the Experience with Left Ventricular Assist Device with Exercise Trial. $J$ Heart Lung Transplant 1999; 18: 1031-40.

13. Humphrey R. Exercise physiology in patients with left ventricular assist device. J Cardiopulm Rehabil 1997; 17: 73-5.

14. Jaski BE, Branch KR, Adamson R, et al. Exercise hemodymanics during long-term implantation of a left ventricular assist device in patients awaiting heart transplantation. J Am Coll Cardiol 1993; 22: 1574-80.

15. Simon MA, Kormos RL, Gorcsan J, et al. Differential exercise performance on ventricular assist device support. $J$ Heart Lung Transplant 2005; 24: 1506-1512.

16. Morrone TM, Buck LA, Catanese KA, et al. Early progressive mobilization of patients with left ventricular assist devices is safe and optimizes recovery before heart transplantation. J Heart Lung Transplant 1996; 15: 423-429.

17. Perme CS, Southard RE, Joyce DL, Noon GP, Loebe M. Early mobilization of LVAD recipients who require prolonged mechanical ventilation. Tex Heart Inst J 2006; 33(2): 130-133.

18. Kennedy MD, Haykowsky M, Humphrey R. Function, eligibility, outcomes, and exercise capacity associated with left ventricular assist devices. Exercise rehabilitation and training for patients with ventricular assist devices. J Cardiopulm Rehabil 2003; 23: 208-17.
19. Grady LK, Meyer PM, Dressler D, et al. Longitudinal change in quality of life and impact on survival after left ventricular assist device implantation. Ann Thorac Surg 2004; 77: 1321-7.

20. Rogers JG, Aaronson KD, Boyle AJ, et al. Continuous flow left ventricular assist device improves functional capacity and quality of life of advanced heart failure patients. J Am Coll Cardiol 2010; 55: 1826-34.

21. Allen JG, Weiss ES, Schaffer JM, et al. Quality of life and functional status in patients surviving 12 months after left ventricular assist device implantation. $J$ Heart Lung Transplant 2010; 29: 278-85.

22. Kugler C, Malehsa D, Tegbur U, et al. Health-related quality of life and exercise tolerance in recipients of heart transplants and left ventricular assist devices: a prospective comparative study. J Heart Lung Transplant 2011; 30: 204-10.

23. Mancini D, Goldsmith R, Levin H, et al. Comparison of exercise performance in patients with chronic severe heart failure versus left ventricular assist devices. Сirculation 1998; 98: 1178-83.

24. de Jonge N, Kirkels H, Lahpor JR, et al. Exercise performance in patients with end-stage heart failure after implantation of a left ventricular assist device and after heart transplantation: an outlook for permanent assisting? J Am Coll Cardiol 2001; 37: 1794-1799.

25. Fink L, Wilson JR, Ferraro N. Exercise ventilation and pulmonary artery wedge pressure in chronic stable congestive heart failure. Am J Cardiol 1986; 57: 249-253.

26. Mettauer B, Geny B, Lonsdorfer-Wolf E, et al. Exercise training with a heart device: a hemodynamic, metabolic, and hormonal study. Med Sci Sports Exerc 2001; 33(1): 2-8.

27. Makita S, Sato S, Sakurada K, Majima M, Kyo S. Rehabilitation for end-stage heart failure patients with LVAS implantation. Comparison of exercise capacity with the patients after coronary artery bypass grafting. Jpn J Card Rehabil 2003; 8(1): 26-28 (in Japanese). 